\title{
Determination of Modal Constant for Fundamental Frequency of Perforated Plate by Rayleigh's Method using Experimental Values of Natural Frequency
}

\author{
Kiran D. Mali and Pravin M. Singru \\ Department of Mechanical Engineering, Birla Institute of Technology and Science, Pilani, K.K. Birla Goa Campus, \\ NH-17B, Zuarinagar, Goa, India- 403726
}

(Received 4 June 2013; accepted 4 March 2014)

In the present work, an expression for the modal constant of the fundamental frequency of the perforated plate was determined experimentally. Rayleigh's formulation was used to calculate the modal constant. The displacement solution was considered to be a linear combination of cosines. In Rayleigh's formulation, fundamental frequency values were taken from experimental analysis. This problem was solved in reverse order by considering known experimental values of the fundamental frequency. Thus, the modal constant expression for fundamental frequency was discovered.

\section{NOMENCLATURE}

$\begin{array}{ll}A & \text { Correction factor } \\ A R & \text { Aspect ratio }=L_{y} / L x,=b / a \\ L_{h} & \text { Centre to centre distance between holes along } \\ & \text { width in mm } \\ L_{v} & \begin{array}{l}\text { Centre to centre distance between holes along } \\ \text { length in mm }\end{array} \\ \left(d+h_{r}\right) & \begin{array}{l}\text { Center to center distance in } \mathrm{mm} \\ \text { Density of material in } \mathrm{kg} / \mathrm{m}^{3}\end{array} \\ D & \text { Diameter of perforation hole in mm } \\ a & \text { Dimension of plate along X axis } \\ b & \text { Dimension of plate along Y axis } \\ L_{x} & \text { Effective plate width in mm } \\ E & \text { Young's modulus in } \mathrm{N} / \mathrm{m}^{2}, \\ L_{Y} & \text { Effective plate length in mm } \\ h & \text { Effective plate thickness in mm } \\ T, u & \text { Kinetic and strain energy of the plate } \\ & \text { respectively } \\ h_{r} & \text { Ligament width in mm } \\ \eta_{l} & \text { Ligament efficiency } \\ M R R & \text { Mass remnant ratio } \\ \omega_{1} & \text { Fundamental frequency of perforated plate } \\ v & \text { in Hz } \\ r & \text { Poisson's ratio } \\ \lambda & \text { Radius of perforation hole in mm } \\ D & \text { Modal constant } \\ & \text { Flexural rigidity of the plate } \\ & =E h^{3} / 12\left(1-v^{2}\right)\end{array}$

\section{INTRODUCTION}

Cutouts are found in mechanical, civil, marine and aerospace structures, commonly as access ports for mechanical and electrical systems or simply to reduce weight. Cutouts are also made to provide ventilation and to modify the resonant frequency of the structures. Perforated plates are often utilized as head plates, end covers, or supports for tube bundles, typically including tube sheets and support plates. Perforated plates are widely used in nuclear power equipment, heat exchangers, and pressure vessels. The holes in the plate are arranged in various regular penetration patterns. Industrial applications include both square and triangular array perforation patterns.

Many researchers have carried out studies of perforated plate structures. Monahan et al. studied the finite element analysis of a clamped plate with different cutout sizes along with experiments using holographic interferometry. ${ }^{1}$ Paramsivam used a finite difference approach in analyzing the effects of openings on the fundamental frequencies of plates with simply supported and clamped boundary conditions. ${ }^{2}$ O'Donnell determined the effective elastic constants for thin perforated plates by equating the strains in an equivalent solid material to the average strains in the perforated material. ${ }^{3}$ Hegarty and Ariman investigated free vibrations of rectangular elastic plate either clamped or simply supported with a central circular hole using the least-squares point-matching method. ${ }^{4}$ Aksu and Ali obtained dynamic characteristics of rectangular plates with one or two cutouts using a finite difference formulation along with experimental verifications. ${ }^{5}$ Ali and Atwal studied the natural frequencies of simply supported rectangular plates and rectangular cutouts using the Raleigh Ritz method. ${ }^{6}$ Reddy studied linear and large amplitude flexural vibration of isotropic and composite plates with cutout by using the finite element method. ${ }^{7}$ Chang and Chiang studied the vibration of a rectangular plate with an interior cutout by using the finite element method. ${ }^{8}$ Lam et al. presented an efficient and accurate numerical method in the study of the vibration of rectangular plates with cutouts and non- homogeneity. ${ }^{9}$ They found the deflection function for the originally complex domain by dividing the problem domain into appropriate rectangular segments. Lam and Hung investigatedflexural vibrations of plates with discontinuities in the form of cracks and cutouts using a scheme that combines the flexibility of dividing the problem domain into appropriate segments and the high accuracy resulting from the use of orthogonal polynomial functions, generated using the Gram-Schmidt process. ${ }^{10}$ Lee et al. predicted the natural frequencies of rectangular plates with an arbitrarily located rectangular cutout. ${ }^{11}$ Mundkur et al. studied the vibration of square plates with square cutouts by using boundary characteristics orthogonal polynomials satisfying the bound- 